\title{
Evaluation of Linear-Load Modelling Effects on Distribution Network Reconfiguration in the Presence of Harmonic Loads
}

\author{
MohamadAli Amini, Alireza Jalilian', Milad Rahimi Pour Behbahani \\ Power Quality Research Lab, Iran University of Science and Technology, Tehran, Iran \\ $\underline{\text { Mo_Amini@elec.iust.ac.ir, Jalilian@iust.ac.ir, Milad_Rahimi@elec.iust.ac.ir }}$
}

\begin{abstract}
Recently, Power Quality is attracting the attention of researchers focused on distribution network reconfiguration. Mainly, harmonic distortion as an emerging issue of distribution networks plays an essential role in power quality-oriented network reconfiguration. Commonly, harmonic analysis of distribution networks is being performed while considering non-linear loads, capacitor banks, subtransmission transformers, and line impedances. However, in harmonic analysis, the presence of linear loads and their effects are being neglected. Consideration of linear loads in harmonic analysis affects the impedance characteristic of the distribution network. Consequently, it changes the total harmonic distortion of bus voltages. In this paper, for the first time, exhaustive research is performed on the effect of linear loads in distribution network reconfiguration by considering five conventional linear load models. The evaluations are conducted on two balanced and unbalanced distribution networks: IEEE 69-bus and 95-bus practical Iranian distribution network using particle swarm optimization aiming to find the minimum total losses of the network. Comparison results confirm that the linear load modelling is very effective in the optimum configuration.
\end{abstract}

Keywords: Distribution Network Reconfiguration, Power Quality, Linear Load, Harmonics.

\section{Introduction}

Nowadays, with the increase in the utilization of non-linear loads in distribution networks, the harmonic distortion of the network has become one of the parameters of the distribution network studies along with other main parameters such as network losses and voltage profiles. Also, another factor that exacerbating the importance of harmonic studies in distribution networks is the increase in the sensitivity of network loads to distortion level of voltage harmonics of the power supply, where it's increase can cause severe disturbances in performance of control and protection equipment. Accordingly, in recent years, many researchers have considered harmonic study in the distribution network studies. Many solutions have been proposed by researchers to control and limit harmonic

' Corresponding Author

School of Electrical Eng. Iran University of Science \& Technology(IUST), Narmak, Tehran 16844, Iran 
emission in distribution networks, including the use of active, passive and hybrid filters, as well as to control the interfaces of the distributed generation units[1-6].

Another solution of harmonic emission control in distribution networks, which recently numerously presented in the literature, is to perform network reconfiguration while considering the total harmonic distortion [7, 8]. Network reconfiguration means changing network configuration to pursue a particular purpose by changing the state of normally open and normally closed switches under network operational constraints.

Reconfiguration of the distribution network is a common issue among the operators of distribution networks, which was first introduced by prof. Merlin et al. aiming to reduce power losses[9, 10]. Afterward, reconfiguration of distribution network is conducted with several goals, including loss reduction[11, 12], load balancing[13, 14], increase in the utilization of distributed generation resources [15], and increase in voltage stability margin [16] and network restoration [17]. In [18], an optimization approach is proposed to improve the unbalance index of distribution network using re-phasing of single-phase customers.

In recent years, with the rise in the importance of power quality in distribution networks, researchers who focused on reconfiguration of the radial distribution network have addressed this issue in their studies. Among the power quality indices, harmonics have been very much considered due to the widespread use of sensitive loads [19, 20]. In [21], the effect of network reconfiguration on the power quality of the distribution network has been investigated. In this study, in addition to network power losses as the primary and predominant objective of network reconfiguration, other objectives include reduction of voltage harmonic distortion, reduction of voltage unbalance in the network buses, and maximization of voltage sag are considered as components of the objective function. In [8], and [21], the network reconfiguration has been utilized to improve the power quality indices such as harmonics and voltage sag along with the reduction of power losses. In [8], the network reconfiguration was carried out while taking into account the THD of bus voltages as well as network losses, harmonic losses and the bus voltages during the faults to check the voltage sag. It has been shown that in distribution networks, reconfiguration can improve network power quality. The effect of harmonic loads on the network reconfiguration problem has been investigated in $[7,23,24]$ concerning total losses, including fundamental and harmonic losses. In [7], three methods have been compared in terms of speed and convergence including discrete Particle Swarm Optimization (DPSO), Imperialist Competitive Algorithm (ICA) and Shuffled Frog Leaping Algorithm (SFLA). The results show that harmonic losses can change the optimum configuration of the network reconfiguration problem. In [22] and [23] the optimization of network configuration is performed in the presence of distributed generations. In [27], reconfiguration was done in order to improve the losses and harmonic distortion of the network buses. In this reference, the capacitor placement was simultaneously investigated with the network reconfiguration, and it is confirmed that the simultaneous network reconfiguration and capacitor 
placement have more significant effect on the improvement of the objectives in comparison with separate optimization. Besides, in [28], the location of a capacitor was simultaneously optimized along with network reconfiguration, taking into account the effect of non-linear loads and their harmonic distortion. In this study, harmonic losses along with fundamental losses are considered as an optimization objective and the harmonic distortion of the bus voltages is considered as a constraint. In [29], in order to solve the optimization problem, in addition to network losses, voltage sag and harmonic distortions are considered as objectives. In this study, the Pareto method is utilized in order to find several optimal points. After solving the problem, optimal points with different values will be available for the operator.

As it is clear from the literature review, power quality and especially harmonics play a significant role in the distribution network studies[24]. In harmonic studies of distribution networks, in addition to the influence of non-linear loads that inject harmonic currents to the network, other equipment such as capacitors and transformers is also effective in determination of impedance characteristic of the network and, consequently, the amount of harmonics emission in the network [25-28]. Along with compensating capacitors and transformers in the network, linear loads also affect the peak value of the impedance characteristic of the network and its frequency. In other words, linear loads change the resonance characteristics of the distribution network. The term linear-loads refers to a group of network loads that, when a sinusoidal voltage feeds them, the current drawn from the network is also sinusoidal, and only at the frequency of supplied voltage. Most urban and commercial loads, induction motors and synchronous motors are samples of the linear load.

Modelling of non-linear loads in harmonic studies is conducted adequately in the literature. This paper is concerned with the modelling of linear loads. Linear loads are a significant component of the system harmonic impedance. They constitute the main element of damping and they may affect the resonance conditions, particularly at higher frequencies[29].

Therefore, linear loads are one of the effective equipment in the emission of harmonics in the distribution network, which can affect the result of harmonic-related optimization problems such as network reconfiguration as well as determining the optimal location and size of the compensating capacitors [24]. In [33], the effect of linear loads modelling on capacitor placement of distribution network has been studied. The results show the important effect of linear loads modelling in the result of the study. So far, the studies of distribution networks reconfiguration have not been considered the effect of linear loads in harmonic calculations. In this paper, for the first time, exhaustive research is performed on the effect of linear loads in the distribution network reconfiguration by considering five conventional linear load models. Briefly, the main contributions of the paper could be listed as follow: 
- Evaluation of the linear-load modelling effect on impedance characteristic of distribution network buses

- Evaluation of the linear-load modelling effect on the harmonic distortion of network voltages

- Evaluation of the linear-load modelling effect on balanced and unbalanced distribution network reconfiguration results considering THD constraint.

The rest of the paper is as follows. Section 2 describes the problem formulation. Section 3 explains the discrete particle swarm optimization. Network modelling and harmonic load flow are described in section 4. Afterward, case studies and simulation results are presented in section 6. Finally, section 6 contains the conclusion of the paper.

\section{Problem Formulation}

In order to evaluate the effect of linear load modelling method on reconfiguration results, an optimization problem is developed, which utilizes six scenarios of linear load models. Each scenario corresponds to one linear load model. Total power losses as the most crucial distribution network operational characteristic are considered as the objective function of the optimization problem. The total power losses of the network consist of both fundamental and harmonic losses of the network as follow [30]:

$$
\text { Objective Function : } P_{\text {Loss }-3 p h}=\sum_{h=1}^{d}\left(\sum_{m=1}^{l}\left(\sum_{j=a}^{c} \operatorname{Re}\left\{\left(V_{j}^{h}(p)-V_{j}^{h}(k)\right) I^{* h}(m)\right\}\right)\right)
$$

where:

$l=$ Number of lines,

$p$ and $k:$ Sending and receiving end of line $l$,

$I^{* h}(m)$ : Current conjugate of $m$ th line between $p$ th node and $k$ th node,

$j=a, b, c$ phases,

$h=h$ th harmonic order of current and voltage, which starts from 1 to maximum studied harmonic order $d$.

For each scenario (each linear load model), the network reconfiguration problem is optimized, and the results are compared.

\section{Optimization constraints:}


In solving the optimization problem of network reconfiguration, some mandatory technical constraints of the network must be considered as follow:

Voltage Constraints: Two sets of constraints are assumed on bus voltages for their RMS and THD[31]. Constraints on RMS values are defined with the lower and upper bounds, $V^{\min }$ and $V^{\max }$ respectively, as follows:

$$
V^{\min } \leq \sqrt{\sum_{h}\left[V_{i}^{(h)}\right]^{2}} \leq V^{\max }, \text { for } i=1, \ldots, n
$$

where $V_{i}^{(h)}$ is the RMS value of the voltage at bus $i$ for harmonic $h$. The voltage distortion constraint is considered by specifying the maximum THD of voltages denoted with $T H D^{\max }$ :

$$
T H D_{i}=\left(\frac{\sqrt{\sum_{h \neq 1}\left[V_{i}^{(h)}\right]^{2}}}{V_{i}^{(1)}} \times 100\right) \leq T H D^{\max } \quad \text { for } i=1, \ldots, n
$$

The bounds for (2) and (3) are specified by the IEEE-519 standard [32], and they are $V^{\min }=0.9 \mathrm{pu}$, $V^{\max }=1.1 \mathrm{pu}$ and $T H D^{\max }=5 \%[33]$.

Current ampacity constraint: The current ampacity of network branches and tie switches are considered as a constraint in optimization procedure as follow:

$$
I_{i} \leq I_{i}^{\max }, \text { for } i=1, \ldots, m
$$

where $I_{i}$ is branch/tie switch current and $I_{i}^{\max }$ is maximum permissible current of corresponding branch/tie switch.

Radial configuration constraint of the distribution network: the topology of the distribution network must keep radial in each iteration of optimization procedure $[34,35]$.

\section{Particle Swarm Optimization}

One of the most intelligent algorithms that is very useful in solving optimization problems is the discrete particle swarm algorithm (DPSO). In 1995 Kennedy and Eberhart [36], established the discrete form of this algorithm for the first time. The algorithm was first used to simulate bird movement, but after simplifying the initial algorithm, it was observed that the algorithm indeed performed an optimization operation and hence could be used to solve other optimization problems.

In the DPSO algorithm, which is inspired by the life of animals' group, especially birds and fishes, in order to solve an optimization problem, a population of candidate responses using a simple formula is randomly assigned to the domain of the problem and aims to determine optimal global solutions. 
In order to find the optimum configuration, tie switches in each configuration are entered into a string as DPSO algorithm inputs (equation (6)). For more explanation, in the first step, all of the network branches are closed. By closing all of the branches, some loops appear in the network. The number of appeared loops is equal to the number of tie switches. Then, one branch is being selected from each loop. Selected branches will form a string, which is called a "position" in DPSO. Each string is a representative of a network configuration. In this study, the number of strings called population is considered as 60 . Each of these configurations has a corresponding velocity as well as a position. The velocity of the particles, as well as the size of the particles, are proportional to the number of tie switches according to equation (6).

$$
\begin{aligned}
& X_{i}=\left(x_{i 1}, x_{i 2}, \ldots, x_{i n}\right) \\
& V_{i}=\left(v_{i 1}, v_{i 2}, \ldots, v_{i n}\right)
\end{aligned}
$$

It is worth mentioning that in the first iteration, the position of the particles is generated randomly, and in subsequent iterations, the position is updated according to the velocity of the particles. At the above two relations, $n$ represents the number of tie switches in the network.

After random configurations were produced, which actually represent the open switches, in each of the configurations, which satisfies the two constraints of radial configuration and supplying all loads, the objective function is calculated. Then, according to the calculated objective function, the particle velocity is calculated at each iteration using (7) [37].

$$
V_{i}^{k+1}=W\left(V_{i}^{k}\right)+C_{1} \text { rand }_{1} \times\left(\text { Pbest }_{i}^{k}-X_{i}^{k}\right)+C_{2} \text { rand }_{2} \times\left(\text { Gbest }^{k}-X_{i}^{k}\right)
$$

Where constants $C_{1}$ and $C_{2}$ are determined by trial and error. In this paper constants $C_{1}$ and $C_{2}$ are tuned in 2.2 and 1.8 , respectively.

The existence of these two functions causes the algorithm to perform more randomly in order to find and evaluate other optimal points of the objective function in addition to searching for local optima. Furthermore, Pbest $t_{i}^{k}$ is the optimum location of $i$ th particle until $k$ th iteration, Gbest ${ }^{k}$ is the optimum location of all group particles until $k$ th iteration. Besides, in the above-mentioned function, $W$ is calculated using (8) [37].

$$
\mathrm{W}=\mathrm{W}_{\max }-\left(\frac{\left(\mathrm{W}_{\max }-\mathrm{W}_{\min }\right)}{\mathrm{It}_{\max }} \times \mathrm{It}\right)
$$

Where in (7) $\mathrm{W}_{\max }$ and $\mathrm{W}_{\min }$ are equal to 0.9 and 0.4, respectively. $\mathrm{It}_{\max }$ is the maximum determined iterations and It is equal to the iteration in which $W$ is calculated. As it is inferred from the formula (7), $\mathrm{W}$ is linearly reducing in the different iterations. The reduction in the value of $\mathrm{W}$ shows that by increasing in iteration number, the particles have more tendency to reach the best position of the function that is detected so far. Excessive reduction in the value of $\mathrm{W}$ in the last iterations, results in 
slowing down the convergence speed. On the other hands, increasing the value of $\mathrm{W}$ will reduce the probability of convergence to the optimal value. Therefore, for more comprehensive search space in initial iterations, $\mathrm{W}_{\max }$ assumed to 0.9 and for more accuracy in last iterations, $\mathrm{W}_{\min }$ assumed to 0.4 . The position of each particle is calculated by (9).

$$
X_{i}^{k+1}=\operatorname{round}\left(X_{i}^{k}+V_{i}^{k+1}\right)
$$

In the above formula, the reason for utilizing "round" function is that the particle location, which actually represents the TS, must be an integer. Figure 1 represents the flowchart of the combination of discrete PSO and network reconfiguration. The maximum iteration in this study is considered 50. Also, scenarios evaluation procedure is also presented in Figure 1.

As shown in the flowchart, after finding the optimum results for each scenario, the network is adopted in the case of linear load model, and its data is considered as the next scenario.

\section{Network Modelling and Harmonic Load Flow}

Harmonic modelling of the network equipment defines the impedance characteristic of the network and consequently, the harmonic distortion of network voltages. In this section, the harmonic models of network equipment are presented.

Linear-load modelling is a crucial element in harmonic propagation and distortion studies, especially in the presence of capacitor banks in distribution network in which harmonic resonance can occur.

Therefore, in harmonic studies, the considered linear-load model will determine the harmonic propagation in the network and constitute the harmonic damping in higher frequencies[38]. Table 1 shows five linear-load models (constant impedance model) which are considered in harmonic load flow studies $[29,39]$. In this paper, comparative studies are conducted to show the sensitivity of network reconfiguration to the selection of linear load model. It is worth mentioning that the introduced linear load models are utilized only in harmonic load flow studies, while in fundamental load flow the constant power model is used. As shown in Table 1, in this paper, five common models of linear-loads are utilized. Liner-load modelling affect the harmonic impedance characteristics of the system and change the harmonic propagation in the network. Therefore, the models mentioned above are considered in the harmonic calculation.

The harmonic modelling approach used in this paper utilizes the fundamental representation of the system harmonic impedance variation at all buses and the execution of harmonic load flow calculations, to determine the voltage distortion status of network buses. It is worth mentioning that more sophisticated models exist for each component. However, their application is usually contradicted 
by the problematic accessibility to reliable data. Following are the fundamental considerations assumed for simplification of harmonic studies:

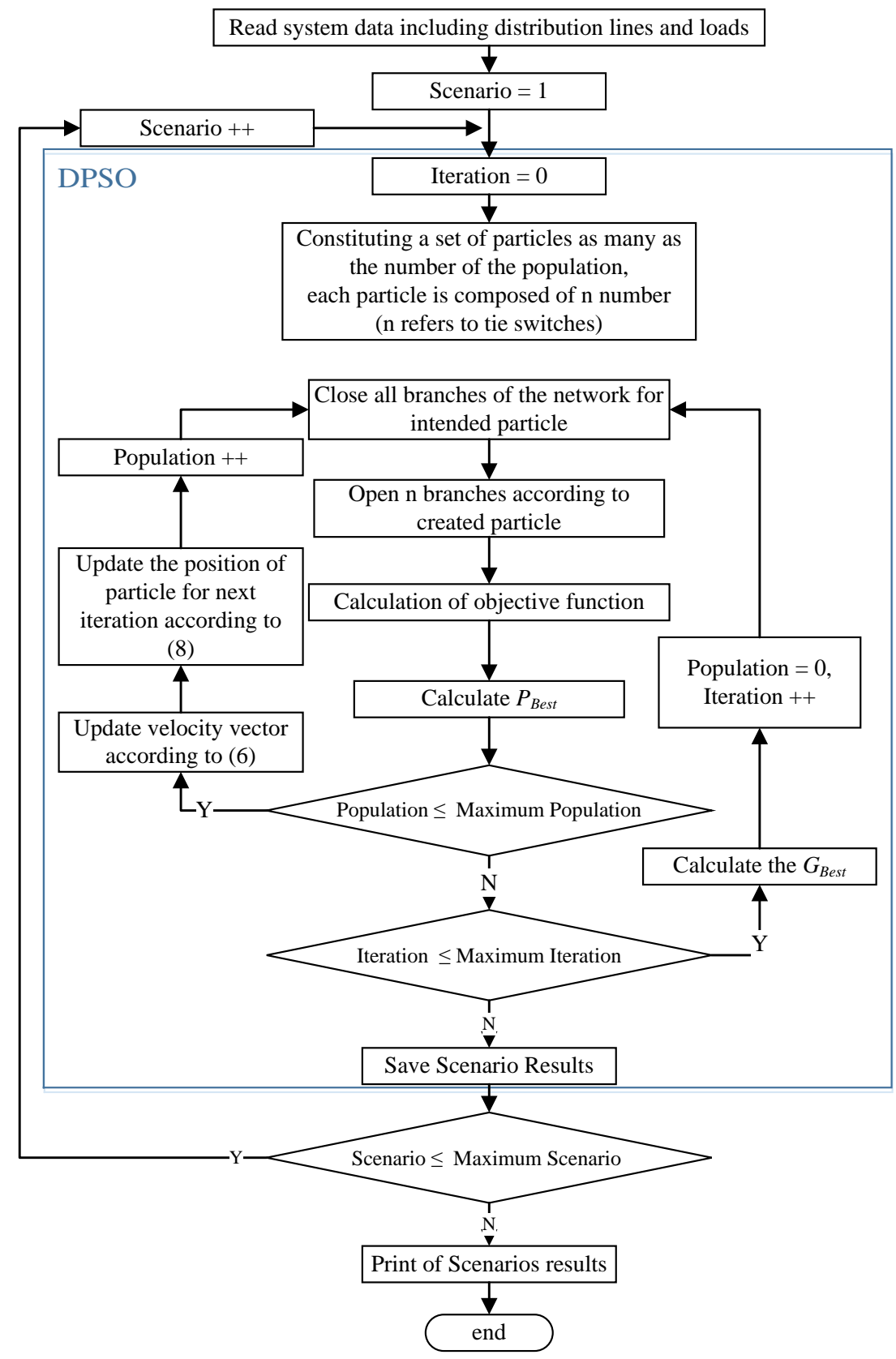

Figure 1: Flowchart of Combination of Discrete PSO and Network Reconfiguration

- Non-linear/harmonic loads are modeled as a current sources in each harmonic order while its magnitude is a function of the fundamental current [7, 8, 40-43].

- Different harmonic orders are assumed to be decoupled. So the fundamental voltages are considered unaffected by harmonic voltages[8].

In this paper, in order to perform exhaustive research on the effect of linear load modelling, the balanced and unbalanced distribution networks are considered. 
Table 1: Linear-Load models

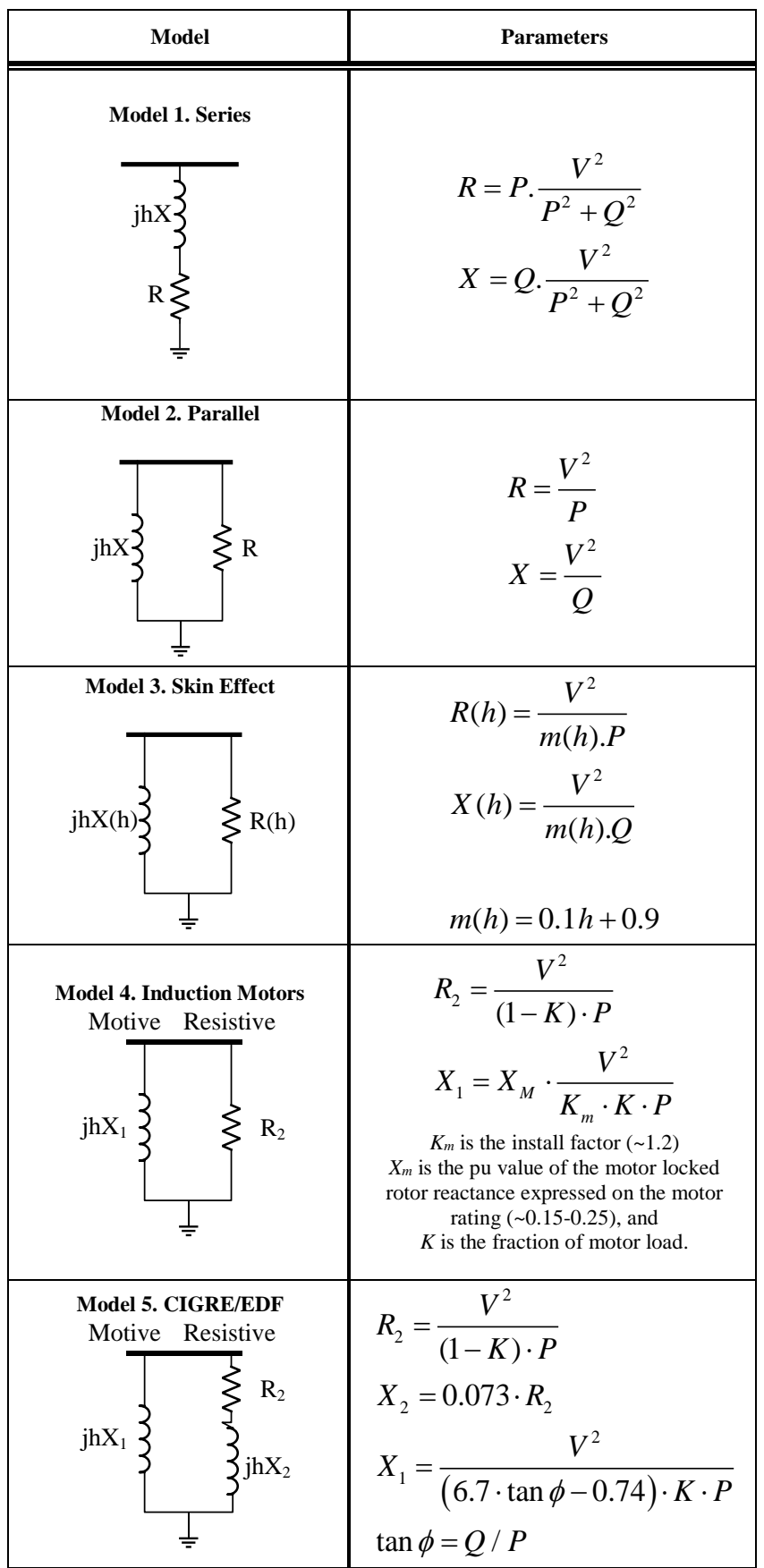

Harmonic load flow of distribution network is performed using Fast Harmonic Load Flow (FHLF) method, which enables the utilization of linear load models and network reconfiguration, simultaneously[44]. FHLF is considering the radial topology of the distribution network and performs harmonic load flow based on the backward/forward sweep method.

Network equipment must be modeled in each harmonic order of harmonic load flow. The line impedance should be calculated by the following equation[44]: 


$$
Z_{L}^{h}=\sqrt{h} R_{L}+j h X_{L}
$$

In addition, the harmonic model of linear loads needs to be calculated based on Table 1, and the corresponding presented formula.

After performing the harmonic load flow, voltage THD at the bus $i$ and power losses $\left(P_{\text {Loss }}\right)$ must be calculated. In this paper, it is assumed that the maximum harmonic order in calculations is 30 . However, there is no limit for harmonic order in the presented method.

\section{Case Studies and Simulation Results}

In order to evaluate the linear load modelling effect on network reconfiguration results, two distribution networks are considered as case study networks as follows:

- IEEE standard 69-bus distribution network,

- Practical Iranian 95-bus distribution network in balanced and unbalanced condition.

First, a well-known IEEE standard 69-bus distribution network is utilized as a benchmark[45]. The base configuration of the system has a single supply point with 69-buses, seven laterals, 68 branches, five loops, or tie switches (switches 69-73), which are normally kept open as shown in dotted lines. The Current ampacity of network branches 1-9 is $400 \mathrm{~A}, 46-49$, and 52-64 is $300 \mathrm{~A}$, and for all other branches, including tie switches are 200 A [45]. The total load for base configuration is 3802.19 $\mathrm{kW}, 2694.6 \mathrm{kVAr}$. The network contains three capacitor banks in different network buses, according to Table 2. Besides, harmonic loads are considered in the network, according to Table 3. Five non-linear load types are assumed in which their harmonic profiles are a function of fundamental current, as presented in Appendix A [46]. Figure 2 shows the single line diagram of the case study network.

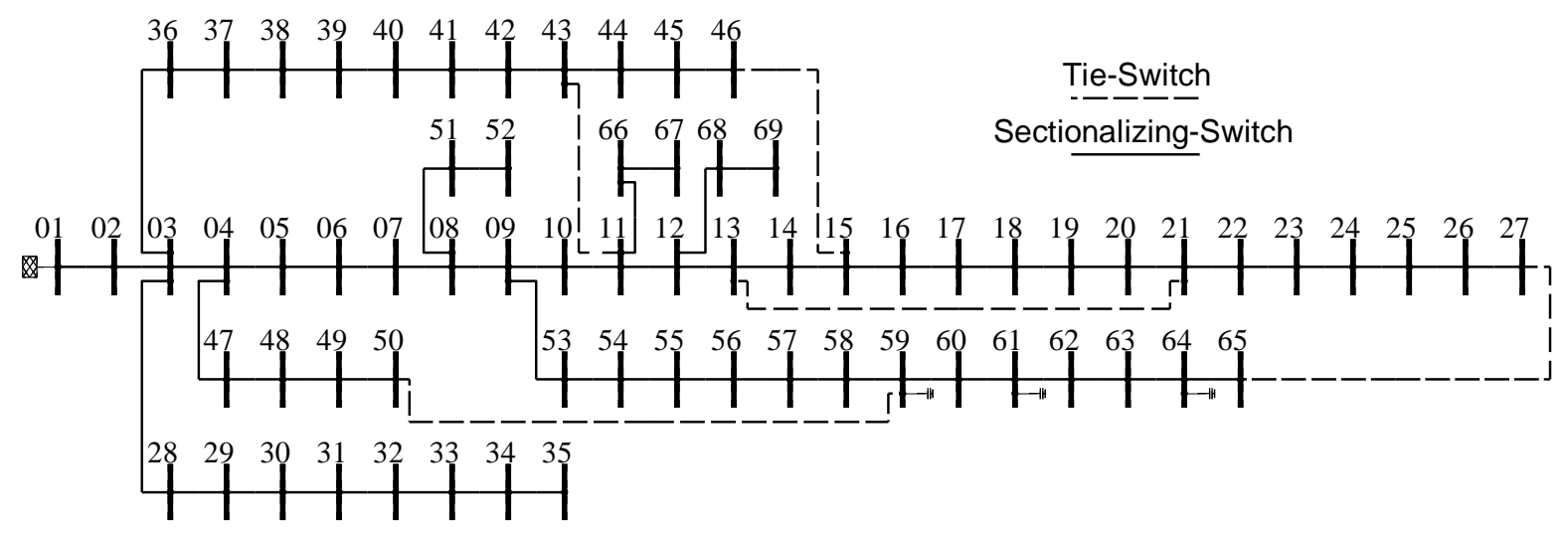

Figure 2: IEEE 69-bus Distribution Network

Table 2: Location of Capacitor Banks

\begin{tabular}{|l|l|l|l|}
\hline Capacitor Bank Size & $\mathbf{7 0 0}$ & $\mathbf{8 0 0}$ & $\mathbf{2 0 0}$ \\
\hline \hline Location & 61 & 64 & 59 \\
\hline
\end{tabular}


Table 3: Location of Non-Linear Loads

\begin{tabular}{|l|l|l|l|l|l|}
\hline Loads & L-1 & L-2 & L-3 & L-4 & L-5 \\
\hline \hline Buses & $14,43,50$ & $10,23,39,41,64$ & $9,21,53$ & $6,37,59,62$ & 33,48 \\
\hline
\end{tabular}

\section{Study Scenarios}

In this paper, six scenarios are devised in order to evaluate the effect of linear load modelling on network reconfiguration. As it is reviewed in the introduction section, it is common in harmonic studies that the linear loads are neglected, and their absorbed current is assumed zero. In this paper, five linear load models, according to Table 1 are considered as five study scenarios. Also, the common no-model method is considered as a benchmark for comparison studies[7]. Therefore, the six scenarios are studied, and results are compared in order to evaluate the effect of linear-load modelling on network reconfiguration results.

The proposed modelling method is utilized to perform the study scenarios. In each scenario, the total power loss is considered as an objective function according to what described before. Table 4, shows the optimization results for six scenarios.

Table 4: Network Reconfiguration Results in six scenarios for 69-bus distribution network

\begin{tabular}{|c|c|c|c|c|c|}
\hline Scenario & Linear load model & Objective function & Opt. Configuration & PLoss & $\boldsymbol{T H D}_{\max }$ \\
\hline \hline Scenario1 [7] & No-load model & Total losses & $\mathbf{1 1 - 1 5 - 6 2 - 7 1 - 7 3}$ & 119.6709 & 4.8323 \\
\hline Scenario2 & Model 1 & Total losses & $\mathbf{1 1 - 1 5 - 6 2 - 7 1 - 7 3}$ & 119.4065 & 4.7888 \\
\hline Scenario3 & Model 2 & Total losses & $\mathbf{1 5 - 5 7 - 6 2 - 7 0 - 7 1}$ & 91.0477 & 4.608 \\
\hline Scenario4 & Model 3 & Total losses & $\mathbf{1 3 - 5 7 - 6 4 - 7 0 - 7 1}$ & 89.7132 & 4.3718 \\
\hline Scenario5 & Model 4 & Total losses & $\mathbf{1 5 - 5 8 - 6 5 - 7 0 - 7 1}$ & 95.1495 & 4.8263 \\
\hline Scenario6 & Model 5 & Total losses & $\mathbf{1 5 - 5 8 - 6 5 - 7 0 - 7 1}$ & 95.4830 & 4.9677 \\
\hline
\end{tabular}

As shown in Table 4 the optimum configurations (corresponds to opened switch of branch number) for six scenarios are different. This is an important point in network reconfiguration, where the method of linear-load modelling affects the optimum configuration. Figure 3 and Figure 4 show the voltage profile and voltage THD of the network buses. 


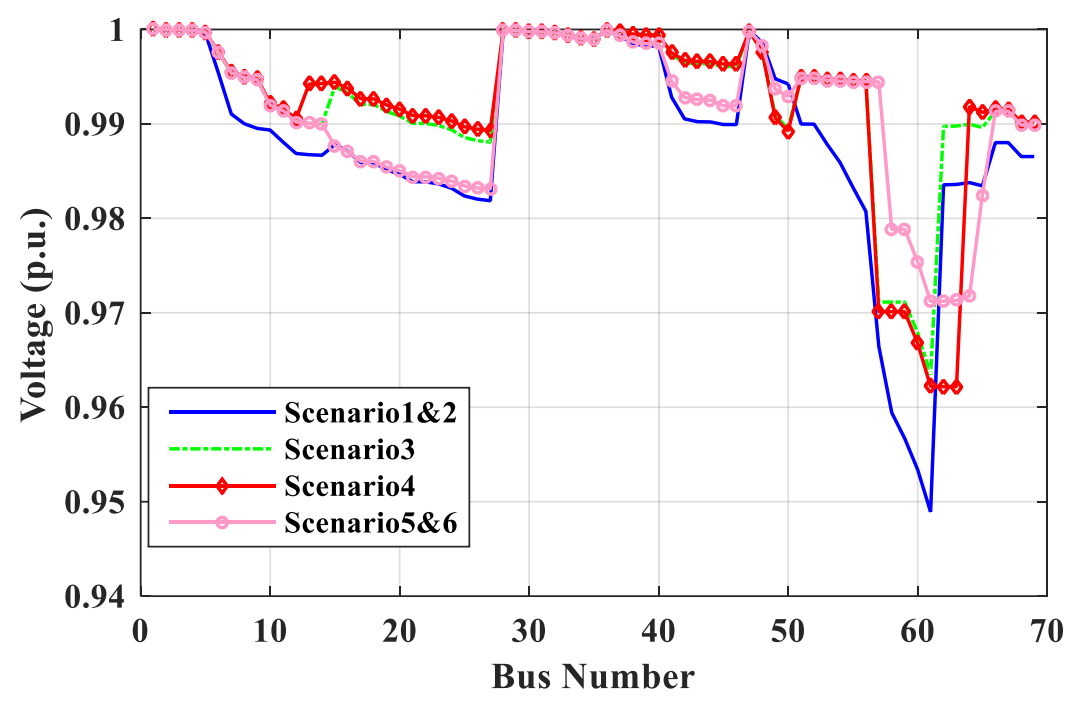

Figure 3: Voltage profile of optimum configuration in six scenarios.

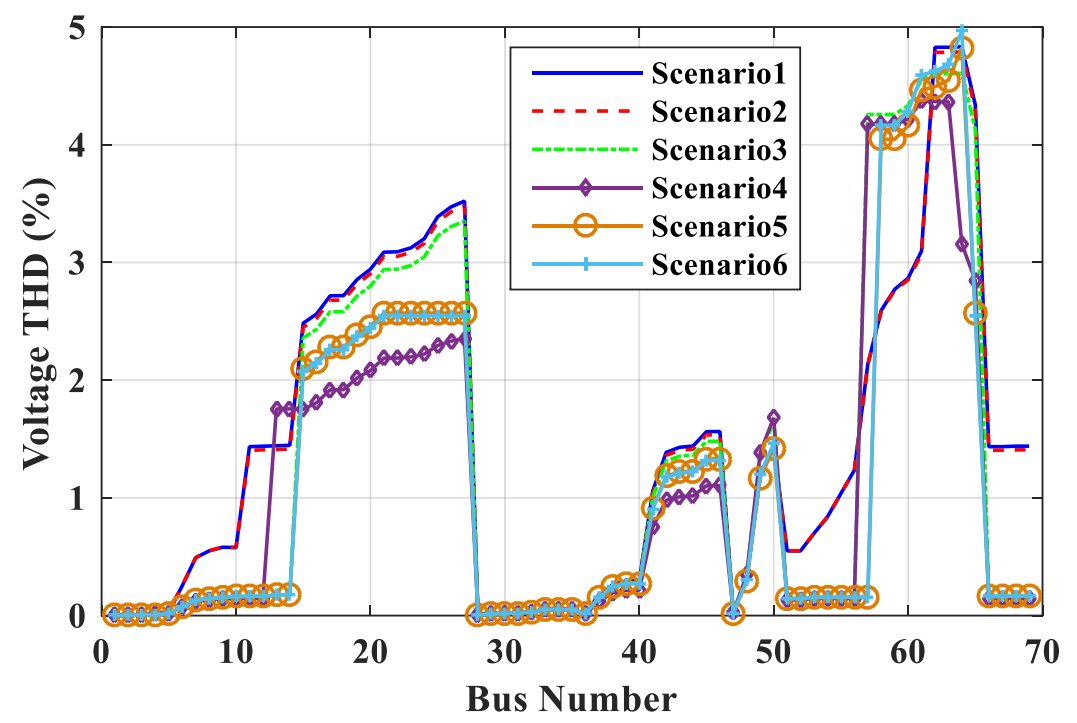

Figure 4: THD profile of optimum configuration in six scenarios.

In addition, the archived optimum configuration of network in the first scenario is not the same configuration in the next ones. 


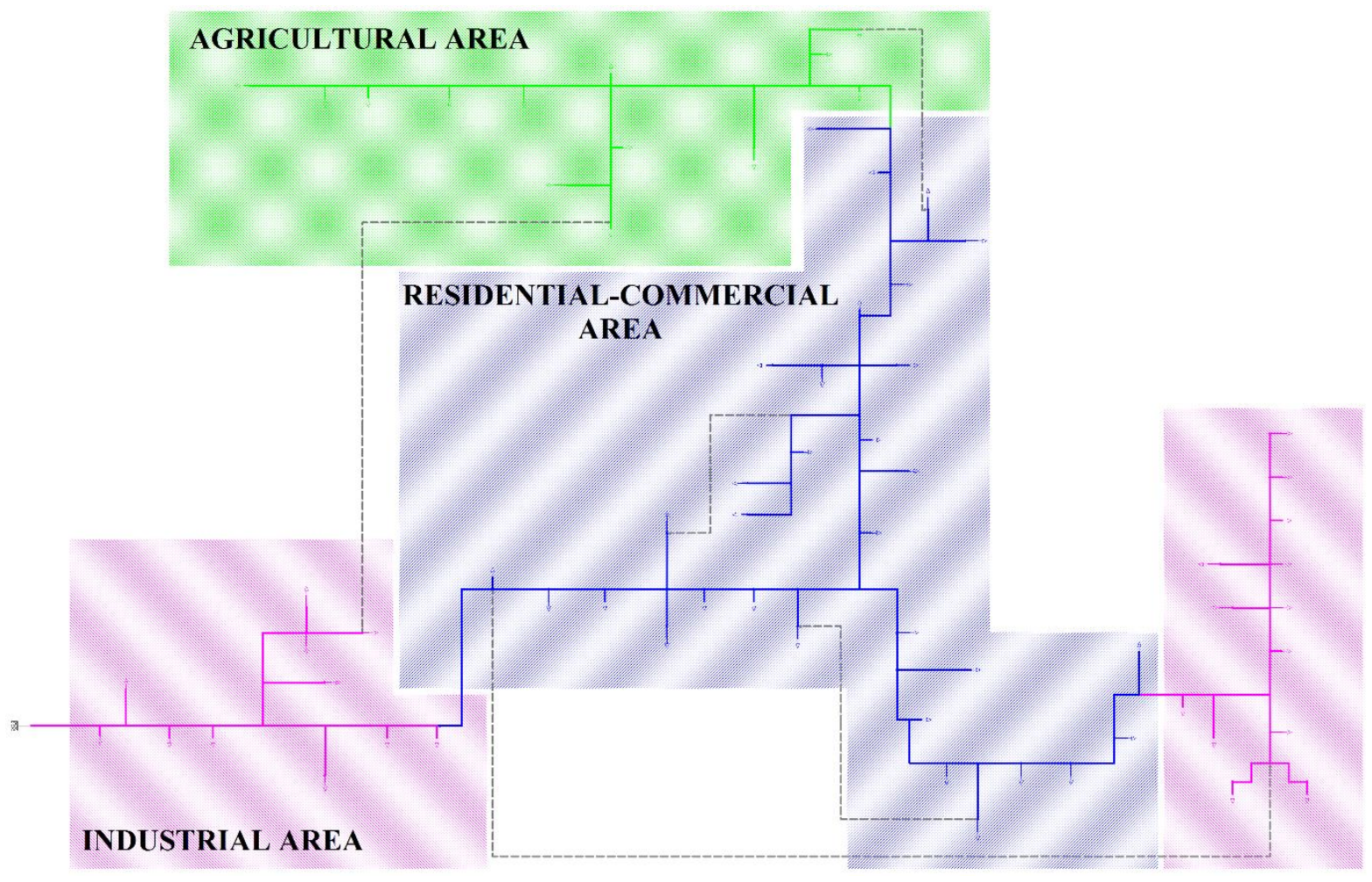

Figure 5: single line diagram of 95-bus Iranian practical distribution network

Therefore, in network reconfiguration in the presence of non-linear loads, disregarding of the linear-loads model, as it is done commonly, degrades the accuracy of the study and will not guarantee the optimum configuration.

In order to evaluate the linear load modelling method in another distribution network, a 95-bus practical Iranian $20-\mathrm{kV}$ distribution network is considered as a case study in balanced and unbalanced conditions [47]. The single line diagram of the network is shown in Figure 5.

- Balanced condition of 95-bus distribution network

The considered practical distribution network is unbalanced inherently. In this section, the unbalanced loads of the network are assumed to be balanced. In the next section, the original form of unbalanced distribution network is considered as a case study. As shown in the Figure 5, the network is a 95-bus radial distribution network, which contains 5 TSs and 94 SSs, respectively. The network voltage is $20 \mathrm{kV}$, and the total load is $6511.5 \mathrm{~kW}$, and 3153.67 kvar. Furthermore, the network contains two capacitor banks, according to Table 5 .

Table 5: Capacitor banks data of 95-bus practical distribution network

\begin{tabular}{|l|l|l|}
\hline Capacitor Bank Size & $\mathbf{8 0 0}$ & $\mathbf{5 0 0}$ \\
\hline \hline Location & 41 & 63 \\
\hline
\end{tabular}


Furthermore, the network divided into three areas according to their dominant categories of costumers. Therefore, the harmonic content of each area is selected based on the harmonic content of the area's consumers as follow:

Table 6: Specifications of harmonic loads of network areas.

\begin{tabular}{|c|c|c|c|c|c|c|}
\hline Area & \multicolumn{3}{|c|}{ Industrial Area } & Residential-Commercial Area & Agricultural-Area \\
\hline \hline $\begin{array}{c}\text { Harmonic } \\
\text { load type }\end{array}$ & Six-Pulse[46] & PWM-ASD[46] & $\begin{array}{c}\text { Six-Pulse } \\
\text { VFD [46 } \\
\text { ] }\end{array}$ & Library-Idle[48] & $\begin{array}{c}\text { Library- } \\
\text { Processing[48] }\end{array}$ & - \\
\hline Location & 5 & $\begin{array}{c}36-40-41-42-46- \\
50\end{array}$ & $10-14$ & $31-35-67-85$ & $17-28-37-59-69$ & - \\
\hline
\end{tabular}

In this paper, the balanced condition of 95-bus practical distribution network is utilized in two evaluations as follow:

- Evaluation of the effect of linear load models on harmonic impedance characteristic of network buses,

- Evaluation of the effect of linear load models on reconfiguration results.

In order to evaluate the effect of linear load models on harmonic impedance characteristic of network buses, the harmonic resonance curve of each bus is defined by its peak impedance value and resonance frequency. Accordingly, the utilization of different linear load models affects these two parameters. In this study, the effect as mentioned above is evaluated in 95-bud practical distribution network in the base configuration. Therefore, based on Table 1, five linear load models in addition to no-load model are considered as six study scenarios. Figure 6 shows the variation of peak impedance value and the resonance frequency of harmonic impedance characteristic of network buses in six scenarios.

As shown in Figure 6, different linear-load models deeply affect the system harmonic impedance peak value in network buses. This issue changes the harmonic distortion in the same situation of nonlinear loads and other network characteristics. For example, the peak value of harmonic impedance characteristic increases from 12.04 in scenario4 to 32.77 in scenario2. This difference is significant in harmonic emission of the network and must be considered in harmonic related studies. However, as shown in Figure 6, the order of harmonic resonance is not affected so much by linear-load consideration.

In order to compare the effect of linear load modelling on the optimum point of reconfiguration problem, six scenarios are considered as a previous case study. Table 7 shows the reconfiguration results for six scenarios.

'Variable Frequency Drive 


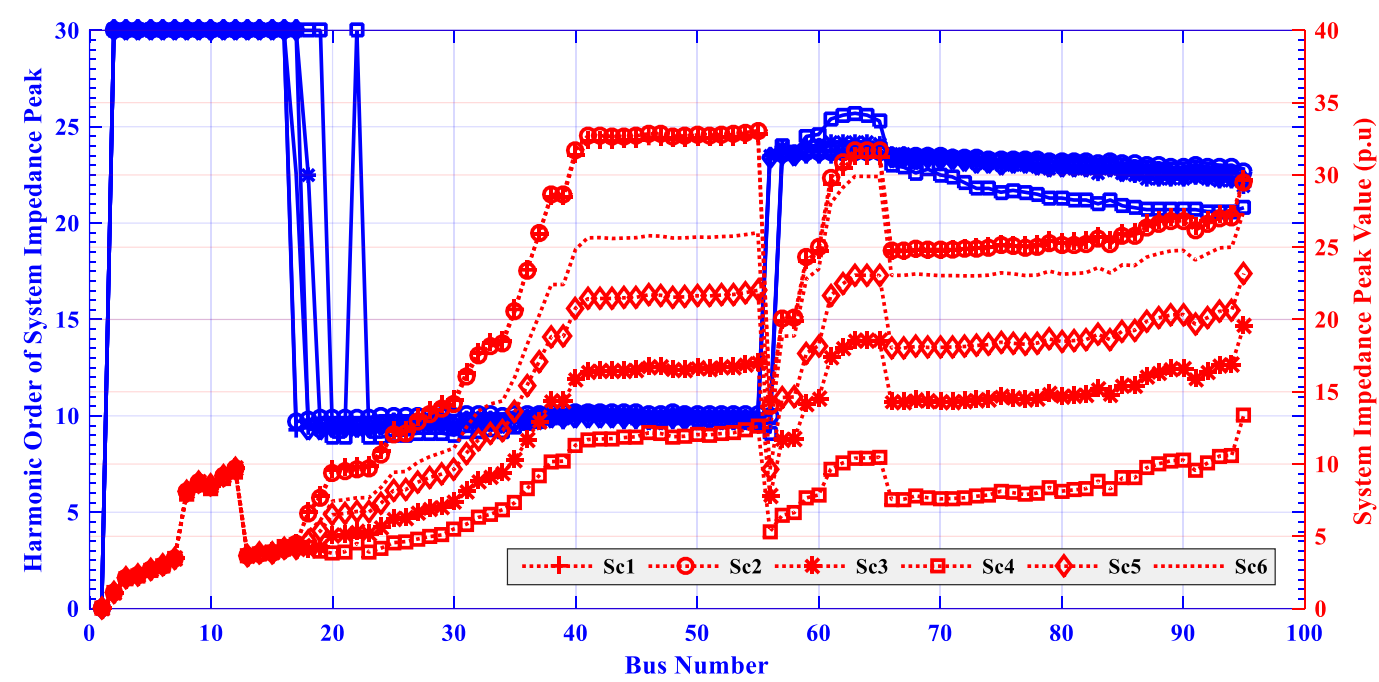

Figure 6: Variation of harmonic impedance characteristic indexes in six scenarios.

Table 7: Network Reconfiguration Results in six scenarios for 95-bus balanced distribution network

\begin{tabular}{|c|c|c|c|c|c|}
\hline \multirow{2}{*}{ Scenario } & \multirow{2}{*}{$\begin{array}{c}\text { Linear load } \\
\text { model }\end{array}$} & \multirow{2}{*}{$\begin{array}{c}\text { Objective } \\
\text { Function }\end{array}$} & $\begin{array}{c}\text { Optimum } \\
\text { Configuration }\end{array}$ & $\begin{array}{c}\text { Total losses } \\
(\mathbf{k W})\end{array}$ & $\begin{array}{c}\text { Maximum THD } \\
(\boldsymbol{\%})\end{array}$ \\
\hline \hline Sc.1 & No-load model & \multirow{5}{*}{ Total Losses } & $24-31-58-77-96$ & 184.0946 & 4.9426 \\
\hline Sc.2 & Model 1 & $24-30-58-76-96$ & 181.9315 & 4.9483 \\
\hline Sc.3 & Model 2 & $24-30-55-72-98$ & 163.2806 & 4.7373 \\
\hline Sc.4 & Model 3 & $24-30-55-72-98$ & 161.0377 & 4.1605 \\
\hline Sc.5 & Model 4 & & $24-30-55-65-75$ & 168.1249 & 4.8814 \\
\hline Sc.6 & Model 5 & & $24-31-58-65-98$ & 170.7549 & 4.9997 \\
\hline
\end{tabular}

As it is shown in Table 7, linear load modelling methods affect the optimization results and the optimum configuration of the network is not the same in six scenarios. Different configurations affect all operational parameters of the network. Figure 7, and Figure 8 show the voltage THD and profile of the network in the optimum configuration of six scenarios.

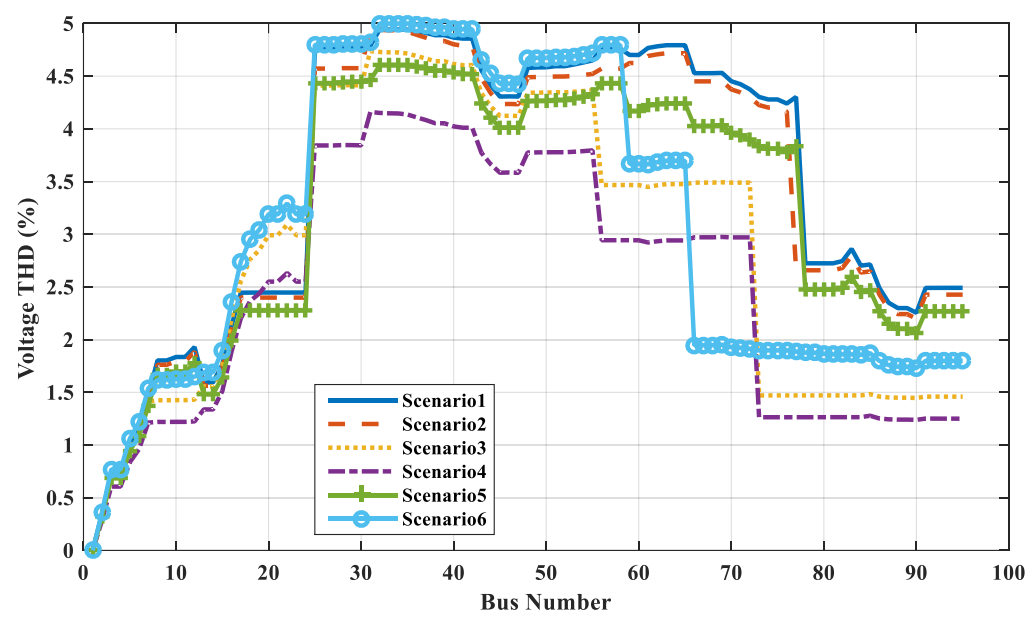

Figure 7: Voltage THD profile of the balanced 95-bus distribution network in optimum configuration of 6 scenarios.

As shown in Figure 7 and Figure 8, in the optimization problems, changing the optimum points, affects the total status of the network. 


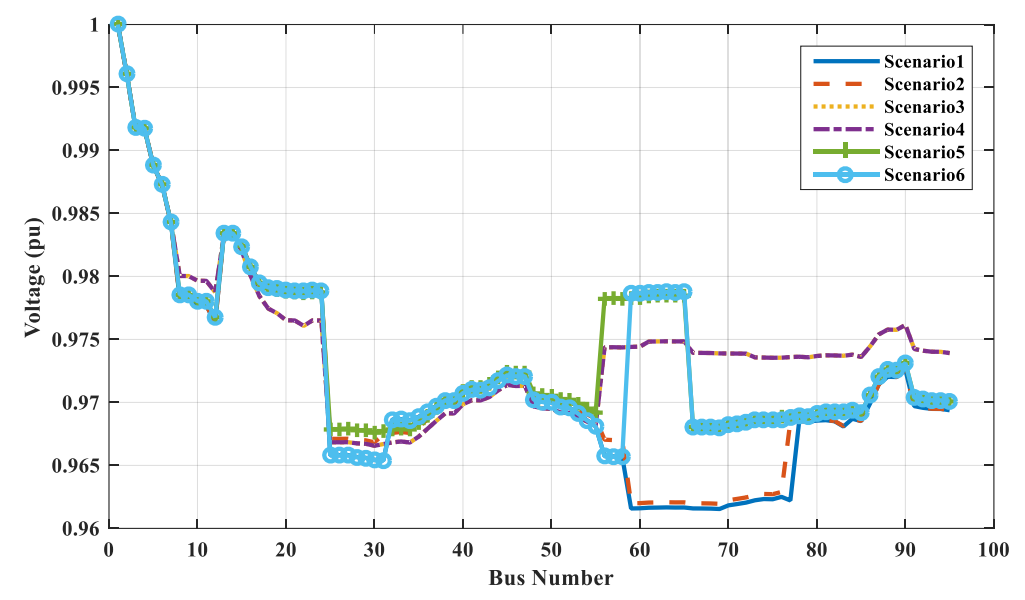

Figure 8: Voltage profile of the balanced 95-bus distribution network in optimum configuration of six scenarios.

- Unbalanced condition of 95-bus distribution network

Inherently, the practical 95-bus distribution network is operating in an unbalanced condition. Therefore, in order to evaluate the effect of linear load modelling in the actual status of distribution networks, the unbalanced condition of the network is considered. Network reconfiguration is performed, aiming to minimize the total power losses of the three phases consisting of fundamental and harmonic losses in phases $\mathrm{a}, \mathrm{b}$ and $\mathrm{c}$ as it is expressed in (1). The previously defined limitations in power, voltage and current is considered as optimization constraints of the problem. Six scenarios consisting of six linear load models, according to table 1 , are evaluated, and the optimization results are presented in table 8.

Table 8: Network Reconfiguration Results in six scenarios for 95-bus unbalanced distribution network

\begin{tabular}{|c|c|c|c|c|c|}
\hline \multirow{2}{*}{ Scenario } & $\begin{array}{c}\text { Linear load } \\
\text { model }\end{array}$ & \multirow{2}{*}{$\begin{array}{c}\text { Objective } \\
\text { Function }\end{array}$} & $\begin{array}{c}\text { Optimum } \\
\text { Configuration }\end{array}$ & $\begin{array}{c}\text { Total losses } \\
(\mathbf{k W})\end{array}$ & $\begin{array}{c}\text { Maximum THD } \\
(\boldsymbol{\%})\end{array}$ \\
\hline \hline Sc.1 & No-load model & \multirow{5}{*}{ Total Losses } & $24-31-58-75-97$ & 186.6358 & 4.577 \\
\hline Sc.2 & Model 1 & $24-30-58-74-98$ & 182.7422 & 4.902 \\
\hline Sc.3 & Model 2 & $24-30-54-73-96$ & 165.0310 & 4.896 \\
\hline Sc.4 & Model 3 & $24-30-55-73-96$ & 165.5626 & 4.549 \\
\hline Sc.5 & Model 4 & & $24-30-55-65-76$ & 171.0080 & 4.242 \\
\hline Sc.6 & Model 5 & & $24-31-58-67-97$ & 172.0751 & 4.496 \\
\hline
\end{tabular}

As it is shown in table 8 , in the unbalanced condition of the distribution network, the optimum configuration is affected by linear load modelling. Therefore, the operational characteristics of the network consisting voltage and THD profile and total network power losses are changed in each scenario. This achievement leads to highlight the effect of linear load modelling in harmonic polluted distribution network studies such as network reconfiguration, capacitor or DG unit placement, etc.

Besides, in this paper, a comparative study is performed considering five different linear-load models. However, a real-world distribution system may have different kinds of linear-loads rather than only one kind of linear-load. Indeed, there exist other issues related to linear load modelling such as improvement of linear load modelling and the methods of effect reduction of linear load, which does 
not fit into the scope of this paper. Despite of simple or complex type of linear load model, the proposed method of this paper is capable of being utilized in accurate distribution network reconfiguration.

In studies of distribution network optimization in the presence of harmonic loads, it is common to consider the following items as object or constraint:

- Maximum allowable THD level as operational constraint,

- Harmonic losses of the network as fraction of total power losses.

As it is shown in the optimization results, the method of linear load modelling affect these two items and, consequently, the optimum point of the problem.

\section{Conclusion}

Harmonic studies of distribution networks are attracted more attention in recent years, including in network reconfiguration. Consideration of the linear-load model in harmonic studies affects the harmonic impedance characteristics of the distribution network and consequently harmonic distortion of network buses. In network reconfiguration, where total losses are mostly considered as a main objective function, harmonic modelling of linear-loads would change the total losses of the distribution networks. In this paper, for the first time, two evaluations are performed; the effect of linear-load modelling on harmonic impedance characteristics of the distribution network and the effect of linearload models on results of network reconfiguration. It is confirmed that the harmonic modelling method of linear-loads would change the optimum configuration of the distribution network. Therefore, in network reconfiguration studies that reflect the presence of non-linear loads, accurate modelling of linear-loads must be considered in order to achieve the real optimum configuration.

\section{References}

[1] W. U. Tareen, S. Mekhilef, M. Seyedmahmoudian, and B. Horan, "Active power filter (APF) for mitigation of power quality issues in grid integration of wind and photovoltaic energy conversion system," Renewable and Sustainable Energy Reviews, vol. 70, pp. 635-655, 2017.

[2] N. C. Koutsoukis, P. S. Georgilakis, and N. D. Hatziargyriou, "Multistage coordinated planning of active distribution networks," IEEE Transactions on Power Systems, vol. 33, pp. 32-44, 2018.

[3] Y. Li, F. Liu, T. K. Saha, O. Krause, and Y. Cao, "Hybrid inductive and active filtering method for damping harmonic resonance in distribution network with non-linear loads," IET Power Electronics, vol. 8, pp. 1616-1624, 2015. 
[4] P. Salmeron and S. P. Litran, "A control strategy for hybrid power filter to compensate four-wires three-phase systems," IEEE Transactions on Power Electronics, vol. 25, pp. 1923-1931, 2010.

[5] Q.-N. Trinh and H.-H. Lee, "An advanced current control strategy for three-phase shunt active power filters," IEEE Transactions on Industrial Electronics, vol. 60, pp. 5400$5410,2013$.

[6] N.-C. Yang and M.-D. Le, "Optimal design of passive power filters based on multiobjective bat algorithm and pareto front," Applied Soft Computing, vol. 35, pp. 257266, 2015.

[7] S. Jazebi, M. M. Hadji, and R. A. Naghizadeh, "Distribution network reconfiguration in the presence of harmonic loads: optimization techniques and analysis," IEEE Transactions on Smart Grid, vol. 5, pp. 1929-1937, 2014.

[8] S. Jazebi and B. Vahidi, "Reconfiguration of distribution networks to mitigate utilities power quality disturbances," Electric Power Systems Research, vol. 91, pp. 9-17, 2012.

[9] M. E. Baran and F. F. Wu, "Network reconfiguration in distribution systems for loss reduction and load balancing," IEEE Transactions on Power delivery, vol. 4, pp. 14011407, 1989.

[10] A. Merlin, "Search for a Minimal-Loss Operating Spanning Tree Configuration for an Urban Power Distribution System," Proc. of 5th PSCC, 1975, vol. 1, pp. 1-18, 1975 1975.

[11] A. A. M. Zin, A. K. Ferdavani, A. B. Khairuddin, and M. M. Naeini, "Reconfiguration of radial electrical distribution network through minimum-current circular-updatingmechanism method," IEEE Transactions on Power Systems, vol. 27, pp. 968-974, 2012.

[12] A. M. Imran and M. Kowsalya, "A new power system reconfiguration scheme for power loss minimization and voltage profile enhancement using fireworks algorithm," International Journal of Electrical Power \& Energy Systems, vol. 62, pp. 312-322, 2014.

[13] M. Farooqi, P. Agarwal, and K. Niazi, "Multi-objective distribution feeder reconfiguration," in Proceedings of the 7th WSEAS/IASME International Conference on Electric Power Systems, High Voltage, Electric Machines, Venice, Italy, 2007, pp. 131-8.

[14] S. Ghasemi, "Balanced and unbalanced distribution networks reconfiguration considering reliability indices," Ain Shams Engineering Journal, 2016.

[15] P. C. Ramaswamy, J. Tant, J. R. Pillai, and G. Deconinck, "Novel methodology for optimal reconfiguration of distribution networks with distributed energy resources," Electric Power Systems Research, vol. 127, pp. 165-176, 2015.

[16] A. Mendes, N. Boland, P. Guiney, and C. Riveros, "Switch and tap-changer reconfiguration of distribution networks using evolutionary algorithms," IEEE transactions on power systems, vol. 28, pp. 85-92, 2013. 
[17] S. Singh, G. Raju, G. Rao, and M. Afsari, "A heuristic method for feeder reconfiguration and service restoration in distribution networks," International Journal of Electrical Power \& Energy Systems, vol. 31, pp. 309-314, 2009.

[18] O. Homaee, A. Najafi, M. Dehghanian, M. Attar, and H. Falaghi, "A practical approach for distribution network load balancing by optimal re-phasing of single phase customers using discrete genetic algorithm," International Transactions on Electrical Energy Systems, vol. 29, p. e2834, 2019.

[19] A. A. Eajal and M. El-Hawary, "Optimal capacitor placement and sizing in unbalanced distribution systems with harmonics consideration using particle swarm optimization," IEEE Transactions on Power Delivery, vol. 25, pp. 1734-1741, 2010.

[20] S. Nesrullah, M. Azah, and S. Hussain, "Reliability improvement in distribution systems employing an integrated voltage sag mitigation method using binary gravitational search algorithm," Journal of Central South University, vol. 20, pp. 30023014, 2013.

[21] A. Dejamkhooy, Y. Fathi Khaneghah, and H. Shayeghi, "Modified branch exchange reconfiguration of active distributed network for simultaneous loss reduction and power quality improvement," International Transactions on Electrical Energy Systems, p. e12065, 2019.

[22] M. Zarei and A. Zangeneh, "Multi-objective optimization model for distribution network reconfiguration in the presence of distributed generations," International Transactions on Electrical Energy Systems, vol. 27, p. e2425, 2017.

[23] K. Hesaroor and D. Das, "Annual energy loss reduction of distribution network through reconfiguration and renewable energy sources," International Transactions on Electrical Energy Systems, p. e12099, 2019.

[24] S. A. Taher, A. Karimian, and M. Hasani, "A new method for optimal location and sizing of capacitors in distorted distribution networks using PSO algorithm," Simulation Modelling Practice and Theory, vol. 19, pp. 662-672, 2011.

[25] S. P. Ang and Z. Wang, Ferroresonance simulation studies of transmission systems: University of Manchester, 2010.

[26] A. Elmitwally and A. Eldesouky, "An approach for placement and sizing of capacitor banks in distribution networks with distributed wind generation," International Transactions on Electrical Energy Systems, vol. 23, pp. 539-552, 2013.

[27] R. Lamedica, A. Ruvio, P. F. Ribeiro, and M. Regoli, "A Simulink model to assess harmonic distortion in MV/LV distribution networks with time-varying non linear loads," Simulation Modelling Practice and Theory, vol. 90, pp. 64-80, 2019.

[28] M. Amini, A. Jalilian, and M. R. P. Behbahani, "A new method for evaluation of harmonic distortion in reconfiguration of distribution network," International Transactions on Electrical Energy Systems, vol. 30, p. e12370, 2020.

[29] R. Burch, G.-k. Chang, C. Hatziadoniu, M. Grady, Y. Liu, M. Marz, et al., "Impact of aggregate linear load modeling on harmonic analysis: a comparison of common practice 
and analytical models," IEEE Transactions on Power Delivery, vol. 18, pp. 625-630, 2003.

[30] M. Rahimi Pour Behbahani, A. Jalilian, and M. Amini, "Reconfiguration of distribution network using discrete particle swarm optimization to reduce voltage fluctuations," International Transactions on Electrical Energy Systems, vol. 30, p. e12501, 2020.

[31] H. Mehrjerdi, R. Hemmati, and E. Farrokhi, "Nonlinear stochastic modeling for optimal dispatch of distributed energy resources in active distribution grids including reactive power," Simulation Modelling Practice and Theory, vol. 94, pp. 1-13, 2019.

[32] "IEEE Guide for Harmonic Control and Reactive Compensation of Static Power Convertors," ANSI/IEEE Std 519-1981, pp. 1-54, 1981.

[33] M. Amini and A. Jalilian, "Modelling and improvement of open-UPQC performance in voltage sag compensation by contribution of shunt units," Electric Power Systems Research, vol. 187, p. 106506, 2020.

[34] S. Esmaeili, A. Anvari-Moghaddam, S. Jadid, and J. M. Guerrero, "Optimal simultaneous day-ahead scheduling and hourly reconfiguration of distribution systems considering responsive loads," International Journal of Electrical Power \& Energy Systems, vol. 104, pp. 537-548, 2019.

[35] M. Amini and A. Jalilian, "Reduction of short-term flicker emission using genetic algorithm-based network reconfiguration in distribution network."

[36] J. Kennedy and R. C. Eberhart, "A discrete binary version of the particle swarm algorithm," in Computational Cybernetics and Simulation, 1997.

[37] J. Olamaei, T. Niknam, and G. Gharehpetian, "Application of particle swarm optimization for distribution feeder reconfiguration considering distributed generators," Applied Mathematics and computation, vol. 201, pp. 575-586, 2008.

[38] P. Ribeiro, "Investigations of harmonic penetration in transmission systems," Ph.D. dissertation, University of Manchester (UMIST), Manchester, U.K., 1985.

[39] S. A. Papathanassiou and M. P. Papadopoulos, "Harmonic analysis in a power system with wind generation," IEEE Transactions on power delivery, vol. 21, 2006.

[40] N.-C. Yang and M.-D. Le, "Three-phase harmonic power flow by direct Z BUS method for unbalanced radial distribution systems with passive power filters," IET Generation, Transmission \& Distribution, vol. 10, pp. 3211-3219, 2016.

[41] A. Bonner, T. Grebe, E. Gunther, L. Hopkins, J. Mahseredjian, N. Miller, et al., "Modeling and simulation of the propagation of harmonics in electric power networks. 2. sample systems and examples," IEEE Transactions on Power Delivery, vol. 11, pp. 466-474, 1996.

[42] A. Bonner, T. Grebe, E. Gunther, L. Hopkins, M. Marz, J. Mahseredjian, et al., "Modeling and simulation of the propagation of harmonics in electric power networks. 1. Concepts, models, and simulation techniques," IEEE Transactions on Power Delivery, vol. 11, pp. 452-465, 1996. 
[43] J. Vuletić and M. Todorovski, "Optimal capacitor placement in distorted distribution networks with different load models using Penalty Free Genetic Algorithm," International Journal of Electrical Power \& Energy Systems, vol. 78, pp. 174-182, 2016.

[44] M. Amini, A. Jalilian, and M. R. P. Behbahani, "Fast network reconfiguration in harmonic polluted distribution network based on developed backward/forward sweep harmonic load flow," Electric Power Systems Research, vol. 168, pp. 295-304, 2019.

[45] J. Savier and D. Das, "Impact of network reconfiguration on loss allocation of radial distribution systems," IEEE Transactions on Power Delivery, vol. 22, pp. 2473-2480, 2007.

[46] F. Sayadi, S. Esmaeili, and F. Keynia, "Feeder reconfiguration and capacitor allocation in the presence of non-linear loads using new P-PSO algorithm," IET Generation, Transmission \& Distribution, vol. 10, pp. 2316-2326, 2016.

[47] S. Jamali and H. Borhani-Bahabadi, "Recloser time-current-voltage characteristic for fuse saving in distribution networks with DG," IET generation, transmission \& distribution, vol. 11, pp. 272-279, 2017.

[48] P. J. Moore and I. E. Portugues, "The influence of personal computer processing modes on line current harmonics," IEEE Transactions on power delivery, vol. 18, pp. 13631368, 2003.

\section{Appendix A. Non-linear Loads Models}

The first assumption of the section 2.2 is about non-linear load modelling. The non-linear or harmonic loads are power electronic based devices (or devices using saturated core) that absorb nonfundamental current when they supplied by pure sinusoidal voltage. The way of non-linear load modelling is by current sources in different harmonic orders according to their harmonic profiles $[5,14$, 20]. Furthermore, the calculation of harmonic content of bus voltages, fundamental voltage and current of loads are calculated. Then using fundamental currents, harmonic current of non-linear loads are determined based on following table. It is assumed that, fundamental bus voltages are assumed to be unaffected by harmonic voltages.

Harmonic spectrums of the non-linear loads [46]

\begin{tabular}{|c|c|c|c|c|c|c|c|c|}
\hline \multicolumn{3}{|c|}{ PWM-ASD } & \multicolumn{3}{c|}{$\begin{array}{c}\text { SIX-PULSE Variable } \\
\text { Frequency Drive }\end{array}$} & \multicolumn{3}{c|}{ SIX- PULSE } \\
\hline $\begin{array}{c}\text { Harmonic } \\
\text { order }\end{array}$ & $\begin{array}{c}\text { Magnitude } \\
(\%)\end{array}$ & Phase $\left(^{\circ}\right)$ & $\begin{array}{c}\text { Harmonic } \\
\text { order }\end{array}$ & $\begin{array}{c}\text { Magnitude } \\
(\%)\end{array}$ & Phase $\left({ }^{\circ}\right)$ & $\begin{array}{c}\text { Harmonic } \\
\text { order }\end{array}$ & $\begin{array}{c}\text { Magnitude } \\
(\%)\end{array}$ & Phase $\left({ }^{\circ}\right)$ \\
\hline $\mathbf{1}$ & 100 & 0 & 1 & 100 & 0 & 1 & 100 & 0 \\
\hline $\mathbf{5}$ & 2 & 0 & 5 & 23.52 & 111 & 5 & 20 & 0 \\
\hline
\end{tabular}




\begin{tabular}{|c|c|c|c|c|c|c|c|c|}
\hline $\mathbf{7}$ & 1.2 & 0 & 7 & 6.08 & 109 & 7 & 14.3 & 0 \\
\hline $\mathbf{1 1}$ & 5.5 & 0 & 11 & 4.57 & -158 & 11 & 9.1 & 0 \\
\hline $\mathbf{1 3}$ & 3.7 & 0 & 13 & 4.2 & -178 & 13 & 7.7 & 0 \\
\hline $\mathbf{1 7}$ & 0.2 & 0 & 17 & 1.8 & -94 & 17 & 5.9 & 0 \\
\hline $\mathbf{1 9}$ & 0 & 0 & 19 & 1.37 & -92 & 19 & 5.3 & 0 \\
\hline $\mathbf{2 3}$ & 0.2 & 0 & 23 & 0.75 & -70 & 23 & 4.3 & 0 \\
\hline $\mathbf{2 5}$ & 0.4 & 0 & 25 & 0.56 & -70 & 25 & 4 & 0 \\
\hline $\mathbf{2 9}$ & 0 & 0 & 29 & 0.49 & -20 & 29 & 3.4 & 0 \\
\hline $\mathbf{3 1}$ & 0 & 0 & 31 & 0.54 & 7 & 31 & 3.2 & 0 \\
\hline
\end{tabular}

